\title{
Juveniles de salmón chinook (Oncorhynchus tshawytscha Walbaum, 1792) en ríos y lagos de la patagonia chilena
}

\section{Chinook salmon juveniles (Oncorhynchus tshawytscha Walbaum, 1792) in rivers and lakes of the Chilean patagonia}

\author{
Jimena Ibarra ${ }^{1 \star}$, Evelyn Habit ${ }^{1,2}$, Ricardi Barra ${ }^{1,2}$ \& Katherin Solís ${ }^{1}$ \\ ${ }^{1}$ Unidad de Sistemas Acuáticos, Centro de Ciencias Ambientales EULA-Chile, Universidad de Concepción. Concepción, \\ Chile. Casilla 160-C. \\ ${ }^{2}$ Centro de Investigaciones en Ecosistemas Patagónicos (CIEP), Coyhaique, Chile. \\ *E-mail: Jimecariola@gmail.com
}

\begin{abstract}
RESUMEN
La presencia de salmón Chinook asilvestrado se ha reportado en Chile desde 1995 con un aumento de la frecuencia de las corridas hacia ríos patagónicos y el consecuente asentamiento temporal de poblaciones juveniles. Con el fin de comprender las implicancias ecológicas de la presencia de estas nuevas poblaciones de juveniles de Chinook en ríos y lagos patagónicos, estudiamos la dieta, condición y edad de individuos de 4 cuencas del sur de Chile (río Puelo, Blanco, Aysén y Futaleufú). Para ello analizamos 104 individuos, 67 provenientes de lagos y 37 provenientes de sistemas fluviales. Algunos juveniles capturados en las cuencas de Aysén (río Aysén) y Futaleufú (lago Yelcho) presentaron edades mayores a 1 año, los cuales corresponden a poblaciones "stream type". Por el contrario, en las cuencas del Blanco, Puelo y Futaleufú (sistemas fluviales) se encontraron juveniles únicamente de edad 0+. Individuos de ambas edades sólo coexistieron en el lago Yelcho. Los individuos de edad 1+ presentaron altos factores de condición y dietas piscívoras, basada en galáxidos nativos. Los individuos menores a 1 año presentaron factores de condición inferiores y una dieta basada principalmente en insectos de origen alóctono. Entre los individuos de edad 0+, sólo aquéllos mayores a 8,5 cm de largo total presentaron peces en sus estómagos. Concluimos que los juveniles de Chinook ingresan a los sistemas patagónicos como depredadores a partir del año de edad, aun cuando si alcanzan tallas mayores a 8,5 cm antes del año también se comportan como ictiófagos. En todos los casos, los peces presa correspondieron a peces nativos. Ello implica una nueva interacción negativa para la fauna íctica nativa patagónica, mediada por el tamaño de los peces introducidos, la cual se manifiesta tanto en depredación como eventualmente por competencia por organismos alóctonos, los cuales son una fuente importante de energía en sistemas oligotróficos como los patagónicos.
\end{abstract}

Palabras clave: Chinook juveniles, dieta, factor de condición, piscivoría, Patagonia, Chile.

\begin{abstract}
The presence of feral Chinook salmon has been reported in Chile since 1995, since when it has been reported an increase in the frequency of runs into rivers of Patagonia and the consequent temporary settlement of juvenile populations. In order to understand the ecological significance of the presence of these new populations of juvenile Chinook in rivers and lakes, we analyze the diet, condition and age of individuals present in four basins of southern Chile (Puelo, Blanco, Aysen and River basins). We analyzed 104 individuals, 67 from 37 from lakes and river systems. Some juveniles caught in the Aysén watershed (river Aysén) and (lake Yelcho) had ages greater than 1 year, which correspond to "stream type" populations. By contrast, in the basins of Blanco, Puelo and Futaleufú (river systems) only age 0 + individuals were found. Juveniles of both ages coexisted only in lake Yelcho. The individuals of age $1+$ showed high condition factors and piscivorous diets, based in native Galaxids. Individuals of less than 1 year showed lower condition factors and diets based mainly on insects of allochthonous origin. Among individuals of age $0+$, only those greater than $8.5 \mathrm{~cm}$ in total length had fish in their stomachs. We conclude that age 1 juvenile Chinook enter Patagonian systems as piscivore predators, and if they reach sizes greater than $8.5 \mathrm{~cm}$ before the completion of the first year they also behave as piscivores. In all cases, prey fish were composed of native fish. This suggests a new negative interaction for the native fish fauna of Patagonia, mediated by the size of exotic fish, such as predation, and possibly also competition for allochthonous organisms, which are an important source of energy in oligotrophic systems as the Patagonian.
\end{abstract}

KEYwords: Chinook juveniles, diet, condition factors, piscivory, Patagonia, Chile. 


\section{INTRODUCCIÓN}

Chinook (Oncorhynchus tshawytscha Walbaum 1792) es un salmónido anádromo nativo del hemisferio norte, desde California hasta Alaska, y de las costas asiáticas del Pacífico norte (Drummond 1982). Durante su etapa adulta habita en el mar (entre 3 a 6 años) hasta alcanzar la madurez sexual, retornando luego al río donde eclosionó para su reproducción. Esta migración, conocida como “corridas o runs”, son masivas y sincrónicas para una cohorte. Generalmente ocurren en otoño o verano, e incluyen individuos que nacieron en el mismo río y algunos extraviados de ríos vecinos (Unwin \& Quinn 1993). Poco después del desove, los machos y las hembras mueren en el lugar, mientras que los embriones se desarrollan en un nido de grava durante dos a seis meses (dependiendo de la temperatura del agua) antes de eclosionar como alevines (Correa \& Gross 2007). Según el tiempo de residencia en los cuerpos de agua dulce, se reconocen dos tipos de poblaciones juveniles, las que migran al océano en su primer año de nacimiento ("oceantype”) y las que migran después de pasar 1 año en agua dulce (“stream-type”) (Healey 1991, Ruiz \& Marchant 2004).

En el sur de Chile, la presencia de individuos asilvestrados de salmón Chinook se ha reportado desde 1995 (Soto 1997) con una tendencia a aumentar la frecuencia de sus corridas y su distribución geográfica (Correa \& Gross 2007), con el consecuente asentamiento de poblaciones juveniles en sistemas límnicos. El incremento de poblaciones asilvestradas con corridas anuales ha sido atribuido tanto a escapes masivos desde centros de acuicultura como a las condiciones abióticas de los ríos (Soto et al. 2001) y a la baja riqueza de especies de peces nativas como posibles competidores o predadores (Campos et al. 1998). Todo lo anterior facilitaría la invasión de especies foráneas, explicando la baja resistencia a la invasibilidad de la Patagonia (Cussac et al. 2004).

Las distintas especies de salmónidos introducidos en la Patagonia representan una amenaza para las especies nativas debido al incremento de los niveles de piscivoría en ríos y lagos (Macchi et al. 1999, Aigo et al. 2008, Milano et al. 2002; Milano et al. 2006, Ortiz-Sandoval 2006). En todos estos casos, las principales especies salmónidas ictiófagas reportadas son las truchas café (Salmo trutta) y arcoíris (Oncorhynchus mykiss). Además de los efectos por depredación se ha reportado que las especies nativas son competitivamente desventajosas frente a los salmónidos (McIntosh 2000), produciéndose el consecuente desplazamiento y disminución de la fauna íctica nativa (Soto et al. 2006, Soto et al. 2007, Habit et al. 2010).

Los efectos que pueden generar las poblaciones del salmón Chinook en los sistemas patagónicos son de distinto origen y magnitud según el estadio de vida que se analice. Por una parte, existen efectos asociados a la corridas de adultos y su subsecuente muerte en ríos de bajo orden, lo cual ha sido relativamente bien estudiado (Naiman et al. 2002, Soto et al. 2006, Soto et al. 2007). Ello genera el transporte de nutrientes marinos hacia los ecosistemas dulceacuícolas, así como la facilitación del incremento de otras poblaciones salmonídeas al proveerlas de una fuente adicional de alimento durante la época de desove. Ambos efectos son indirectos, ya que tanto los machos como las hembras dejan de alimentarse al final de su corrida migratoria (Drummond 1982). Sin embargo, la invasión de estos salmones implica también el establecimiento por uno o dos años de nuevos integrantes de las comunidades ícticas patagónicas, correspondientes a los juveniles, de los cuales se tienen menos antecedentes. Con el fin de comprender el significado ecológico de la presencia de estas nuevas poblaciones de juveniles de Chinook en ríos y lagos patagónicos, se analizó la estructura de edades, dieta y factor de condición de individuos provenientes de 4 cuencas del sur de Chile (río Puelo, Blanco, Aysén y Futaleufú). Postulamos que la buena condición aparente en juveniles de estas poblaciones de salmón Chinook podría deberse a que, a partir del año de edad, poseen una dieta ictiófaga basada en peces nativos. Ello implicaría que la especie $O$. tshawytscha ingresa a los sistemas patagónicos como un nuevo depredador de especies nativas.

\section{MATERIALES Y MÉTODOS}

ÁREA DE ESTUDIO

Se analizaron muestras de peces provenientes de 4 cuencas del sur de Chile; cuencas del río Puelo (41 393'11,7'S/

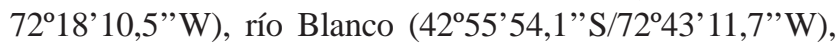
Futaleufú (42058'04,0”S/7243'35,2”W) y Aysén

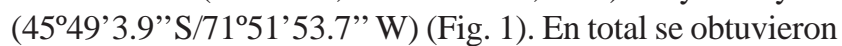
juveniles de $O$. tshawytscha de un total de 6 sitios, 4 en ríos y 2 en lagos. Los sitios de muestreo por cada cuenca, fecha de muestreo y arte de pesca utilizado se describen en la Tabla 1.

Se obtuvo un total de 104 juveniles de Chinook, 67 provenientes de lagos y 37 provenientes de sistemas fluviales. Todos los ejemplares capturados fueron medidos (longitud total y estándar mediante ictiómetro) y pesados (balanza O’Haus de 0.01g de precisión) in situ. Posteriormente fueron procesados y fijados en alcohol al 96\%. A modo de determinar la estructura de edades de los individuos capturados, se realizó la lectura de microincrementos en otolitos Sagittae siguiendo el protocolo de Neilson \& Geen (1982). Para el cálculo del factor de condición se usó la ecuación modificada del método de Fulton (1902),

$$
\mathrm{K}=(\mathrm{W}) / \mathrm{L}^{3} \mathrm{X} 10^{5}
$$


donde W corresponde al peso total del individuo expresado en gramos y L es longitud total en cm. Con el fin de analizar la relación entre factor de condición y las tallas (longitud total) se realizó una regresión simple (StatSoft, Inc. 2004; STATISTICA data analysis software system, version 7.0. www.statsoft.com). Finalmente, para comparar los factores de condición entre cuencas y sistemas fluviales y lacustres, se utilizó un análisis de varianza no paramétrico de KruskalWallis, dado que los datos no cumplieron con los supuestos de normalidad.
El análisis de composición dietaria se realizó mediante el estudio de contenidos estomacales bajo estereomicroscopio, identificando los ítemes presas hasta el mínimo nivel taxonómico posible. Para determinar el aporte numérico y gravimétrico de los diferentes ítemes alimentarios, éstos se agruparon según su origen en 3 categorías: Autóctono, que incluye los ítemes presa constituidos por invertebrados acuáticos no alados; Alóctono, correspondientes a invertebrados terrestres y acuáticos alados (imagos) y el ítem Peces. Se utilizó esta categorización para representar

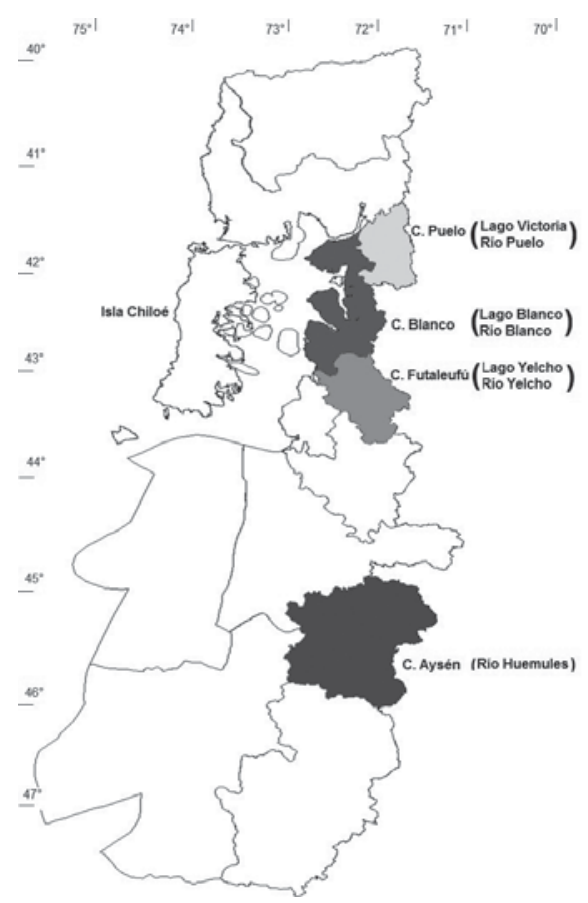

Figura 1. Mapa de las cuencas incluidas en este estudio y sitios de muestreo (ríos/lagos).

FIGURE 1. Map of basins included in this study and sample sites (rivers/lakes).

TABLA 1. Sitios de muestreo y arte de pesca utilizado por cuenca.

TABLE 1. Sampling sites and gear used per basin.

\begin{tabular}{|c|c|c|c|}
\hline Cuenca & Río/Lago & Fecha & Método de Pesca \\
\hline Río Puelo & Lago Victoria & Marzo 2008 & Red de arrastre y Agalleras. \\
\hline (41'39'11,7”S/72²18'10,5”W) & Río Puelo & Marzo 2008 & Red de arrastre y Agalleras. \\
\hline $\begin{array}{c}\text { Río Blanco } \\
\left(42^{\circ} 55^{\prime} 54,1^{\prime \prime} \mathrm{S} / 72^{\circ} 43^{\prime} 11,7^{\prime \prime} \mathrm{W}\right)\end{array}$ & Río Blanco & Marzo 2008 & Red de arrastre y Pesca Eléctrica Halltech \\
\hline Río Futaleufú & Lago Yelcho & Marzo 2008 & Red de arrastre y Pesca Eléctrica Halltech \\
\hline$\left(42^{\circ} 58^{\prime} 04,0 ” S / 72^{\circ} 43^{\prime} 35,2^{\prime \prime} \mathrm{W}\right)$ & Río Yelcho & Marzo 2008 & Red de arrastre y Pesca Eléctrica Halltech \\
\hline 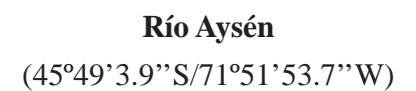 & Río Huemules & Abril 2008 & Red de arrastre y Pesca Eléctrica Halltech \\
\hline
\end{tabular}


los distintos hábitos alimentarios de juveniles de Chinook. A continuación se construyó una matriz de abundancia de ítemes alimentarios para cada muestra (expresada como número de presas por estómago de cada ítem) y otra de biomasa, considerando a cada uno de los individuos analizados como una réplica de un mismo sitio, y a los ítemes presa como las n variables.

La composición dietaria de los juveniles de Chinook fue analizada mediante métodos multivariados a través de las cuencas hidrográficas y sistemas de procedencia de los peces (sistemas lacustres versus sistemas fluviales). Para ello los datos de abundancia de ítem presas fueron transformados mediante raíz cuarta para reducir el peso de los ítemes dominantes y luego se construyó una matriz de similitud basada en el índice de Bray-Curtis (Bray \& Curtis 1957), utilizando el programa PRIMER v.6.15 (Clarke \& Gorley 2005). Se efectuaron análisis exploratorios de los patrones de similitud dietaria entre cuencas y sistemas mediante métodos de cluster y/o análisis de escalamiento multidimensional no métrico (nMDS) para posteriormente poner a prueba las diferencias significativas con la prueba de ANOSIM (Clarke et al. 2005). A modo de determinar el ítem alimentario que presenta una mayor contribución a la similitud de dietas entre edades, se realizó un análisis de similitud porcentual SIMPER de una vía para la composición numérica de la dieta.

\section{RESULTADOS}

De los 104 individuos analizados, 30 correspondieron a ejemplares de más de un año de edad (1+), mientras que los restantes fueron de edad 0+ (Fig. 2). En ríos de la cuenca del Aysén sólo se encontraron individuos de edad 1+, en tanto que los ejemplares de ríos y lagos de las cuencas del Blanco y Puelo sólo correspondieron a individuos de edad 0+ (Fig. 3). En la cuenca del río Futaleufú se encontraron individuos de ambas edades, pero con una mayor frecuencia de peces menores a 1 año. En esta cuenca, todos los individuos 1+ provenían del lago Yelcho. Consistentemente con la edad, los ejemplares de mayor talla se encontraron en las cuencas del Aysén y Futaleufú (> $11 \mathrm{~cm} \mathrm{LT)}$ ), todos ellos de edad 1+ (Fig. 4). Los individuos de edad $0+$ presentaron tallas que van de los 8,8 a 12,5 cm de largo, mientras que los Chinook de edad 1+ presentaron un rango de tallas entre los 11,0 a $14,0 \mathrm{~cm}$ de largo.

El factor de condición se correlacionó leve pero significativamente con la talla $(r=0,21 ; p=0,025)$ y presentó diferencias significativas entre individuos provenientes de los distintos sistemas (Kruskal-Wallis, $\mathrm{H}_{67,61}=0,005 ; \mathrm{p}=0,0000$ ) (Fig. 5). Los individuos provenientes de la cuenca del Aysén y lago Yelcho (cuenca del río Futaleufú) presentaron los mayores valores de factor de condición consistentemente con la presencia de individuos de edad $1+$.

El 97\% de los estómagos analizados presentaron contenido estomacal. En éstos se identificó un total de 54 ítemes dietarios distribuidos en 17 Ordenes y 5 Clases, siendo las más abundantes Coleoptera, Diptera e Himenoptera (Tabla 2). A partir del análisis de contenido estomacal de los juveniles capturados en las cuatros cuencas, se contabilizó un total de 11.254 presas correspondientes a 54 ítemes alimentarios. Del total de presas, 74,74\% fueron ítemes alóctonos, 24,93\% de origen autóctono y 0,33\% ítemes presa peces. El ítem peces estuvo constituido exclusivamente por ejemplares del género Galaxias. Al comparar el aporte promedio de la abundancia de cada tipo de ítem (Alóctono, Autóctono y Peces) entre las diferentes cuencas (Fig. 6), se observa que en promedio para Futaleufú el mayor aporte lo realizan las presas alóctonas y el menor aporte corresponde al ítem peces. Este mismo patrón se observa en la cuenca de Aysén, aunque con una menor diferencia en el aporte entre ítemes alóctono y autóctono. En las cuencas de Puelo y Blanco no se registraron peces como presa y los aportes numéricos de los ítemes alóctonos y autóctonos fueron similares en cada cuenca.

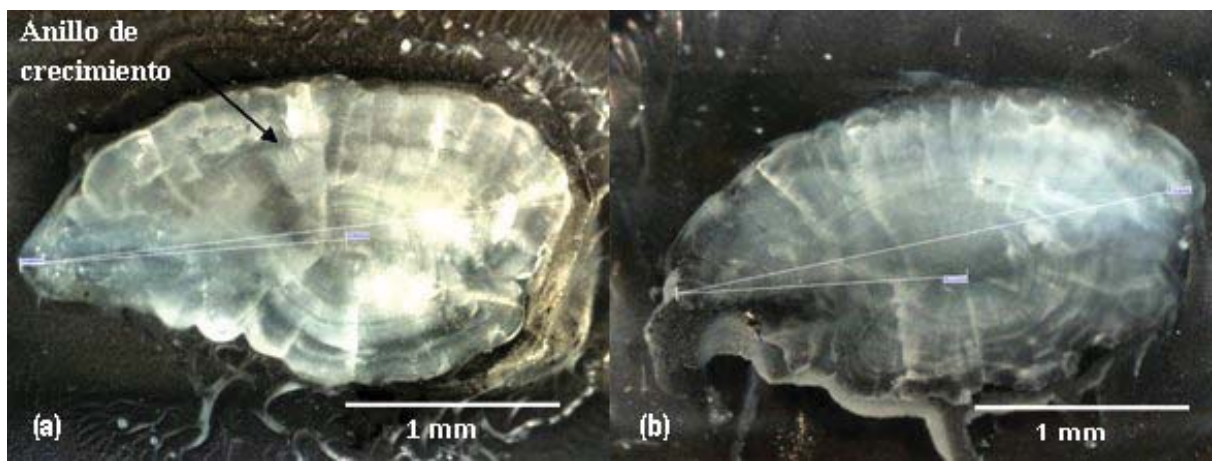

Figura 2. Otolito Sagita de Chinook juveniles. (a): Otolito con 1 anillo de crecimiento anual. (b) Otolito sin anillos de crecimiento anual.

Figure 2. Sagitta otolith of juvenile Chinook. (a): Otolith ring of one year. (b) Otolith with no annual growth rings. 
De acuerdo al aporte gravimétrico, la proporción de los ítemes alimentarios según su origen fue diferente a la numérica. Las presas alóctonas correspondieron al $47,43 \%$, las de origen autóctono a un $14,74 \%$ y los peces $37,83 \%$. En individuos provenientes de las cuencas Blanco y Puelo, el mayor aporte promedio correspondió a ítemes alóctonos, los que representaron casi la totalidad del contenido estomacal en términos de masa (Fig. 6).

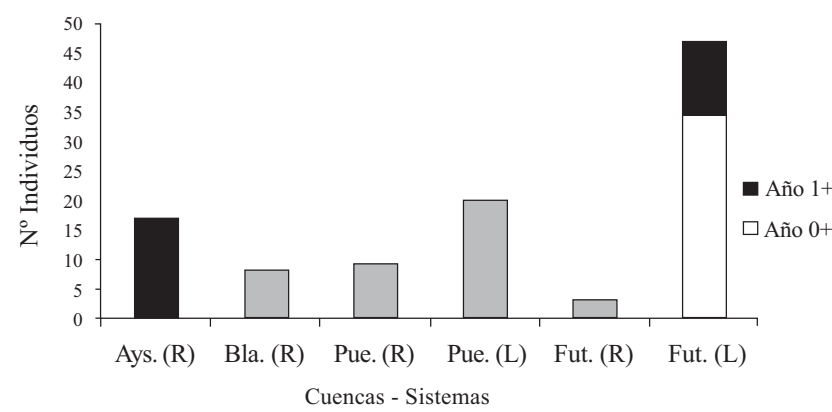

Figura 3. Número de individuos de edad mayor a 1 año (1+) y menor de 1 año $(0+)$ representados en la muestra según cuenca hidrográfica y sistema Lago (L) o Río (R). Ays: Aysén, Bla: Blanco, Pue: Puelo, Fut: Futaleufú.

FIgure 3. Number of individuals older (1+) and younger ( $0+$ ) than one year by watershed and system is lake and $\mathrm{R}$ represents rivers. Ays: Aysén, Bla: Blanco, Pue: Puelo, Fut: Futaleufú.

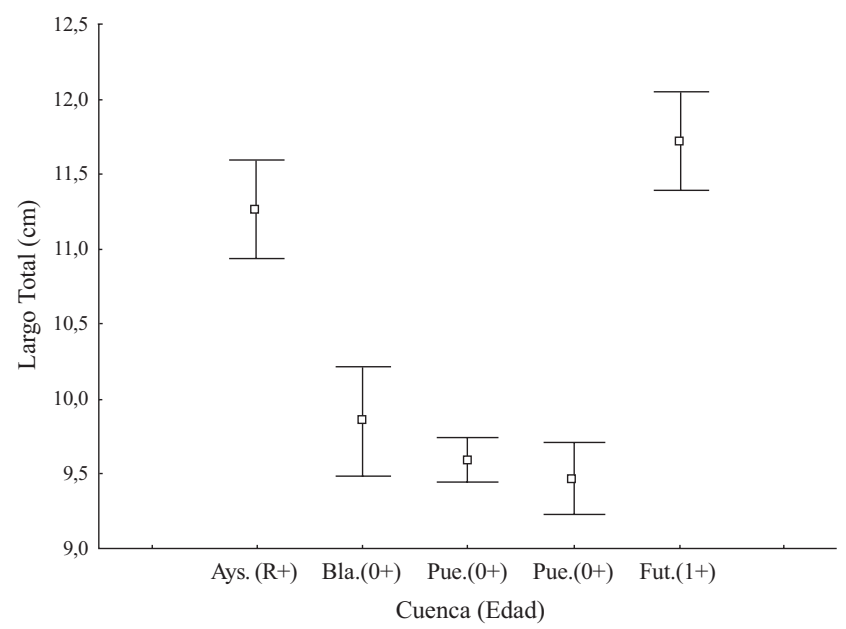

Figura 4. Longitud total promedio ( \pm 1 error estándar) de Chinook juveniles según la cuenca de procedencia y edad $(0+$ y $1+)$. Pue: Puelo, Fut: Futaleufú, Ays: Aysén, Bla: Blanco.

Figure 4. Average total length of juvenile Chinook by watershed and age $(0+$ and $1+)$, using one standard error as a measure of deviation. Pue: Puelo, Fut: Futaleufú, Ays: Aysén, Bla: Blanco.
En tanto, en estómagos de juveniles provenientes de las cuencas de Aysén y Futaleufú, se encontró un aporte promedio similar de los organismos alóctonos y peces, los cuales corresponden a los ítemes principales en término de biomasa. La composición dietaria fue significativamente diferente entre cuencas y entre ríos y lagos (sistemas) tanto para la composición numérica (ANOSIM, Cuencas, $\mathrm{R}=0,644$, $\mathrm{p}=0,001$; Sistemas $\mathrm{R}=0,685, \mathrm{p}=0,001$ ) como para la biomasa por ítemes dietarios (ANOSIM, Cuenca, $\mathrm{R}=0,484, \mathrm{p}=0,001$; Sistemas $\mathrm{R}=0,427, \mathrm{p}=0,001$ ).

Se encontró una alta disimilitud (82,71\%) entre la dieta de los individuos de edad (1+) y peces menores a un año de edad (0+), (Tabla 3). Esta se explica principalmente por el ítem alimentario Trichoptera (adulto), el cual contribuye con un $12,8 \%$ a la disimilitud de dietas entre las edades. Además, este ítem aporta un 35,4\% a la dieta de individuos $0+$, mientras que para los juveniles de edad $1+$ el mayor aporte a la dieta lo entrega el ítem Leptophlebiidae (Adulto) con una contribución de 27,3\%. Ambos itemes son de origen alóctono. Los individuos que consumen el ítem peces en su dieta presentaron tanto edades $0+$ como $1+$. A su vez, el mayor consumo de peces (Galaxias sp.) se presentó en lagos de la cuenca del Futaleufú, mientras que en menor medida se observa un consumo de este ítem en ríos de la cuenca de Aysén.

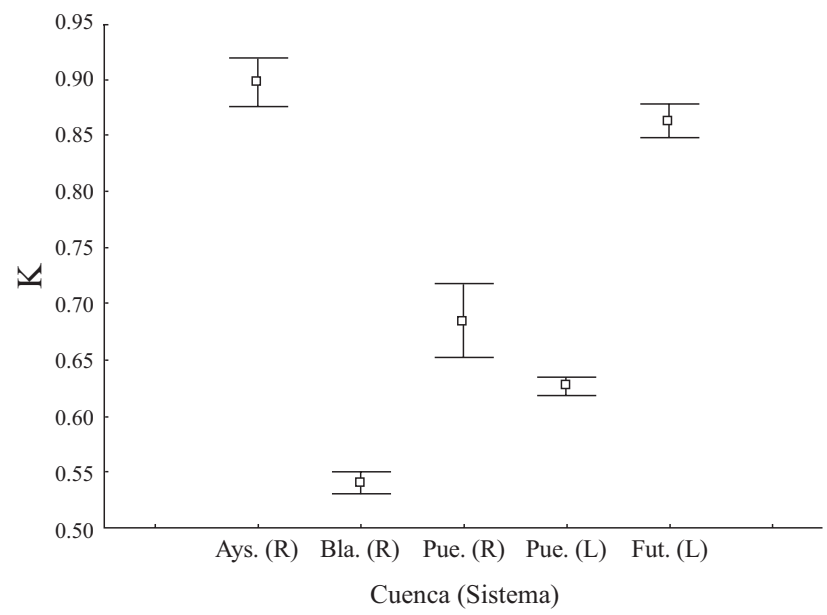

Figura 5. Comparación del factor de condición (K) de Chinook juveniles entre distintas cuencas y sistemas de lago (L) o río (R). Promedio \pm 1 error estándar. Ays: Aysén, Bla: Blanco, Pue: Puelo, Fut: Futaleufú.

Figure 5. Comparison between condition factor (K) of different basins and systems lake (L) or river (R). \pm one standard error. Ays: Aysén, Bla: Blanco, Pue: Puelo, Fut: Futaleufú. 
TABLA 2. Ítemes consumidos por los peces capturados en las cuatro cuencas muestreadas.

TABLE 2. Items consumed by the fish caught in the four basins sampled.

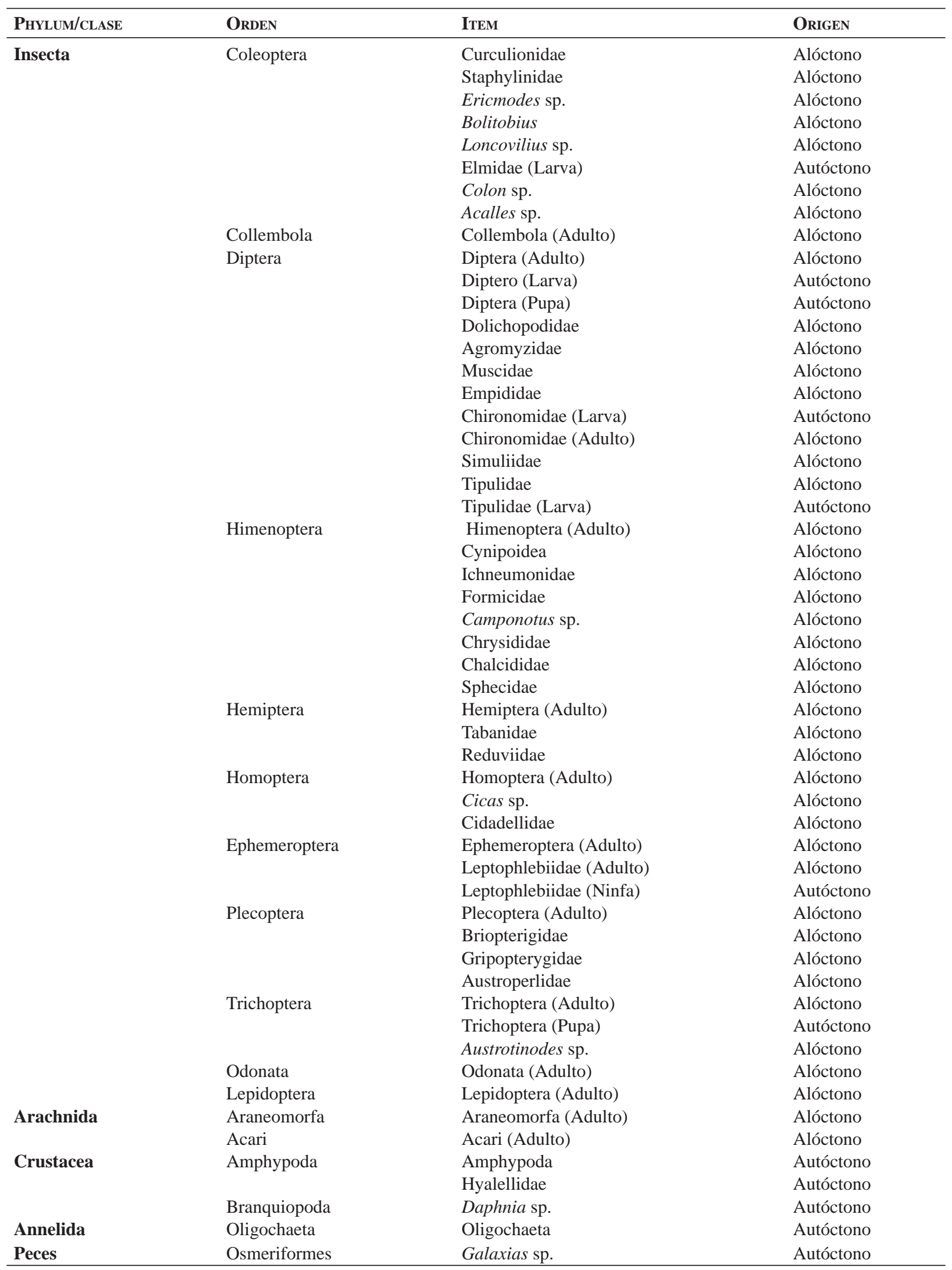


a)

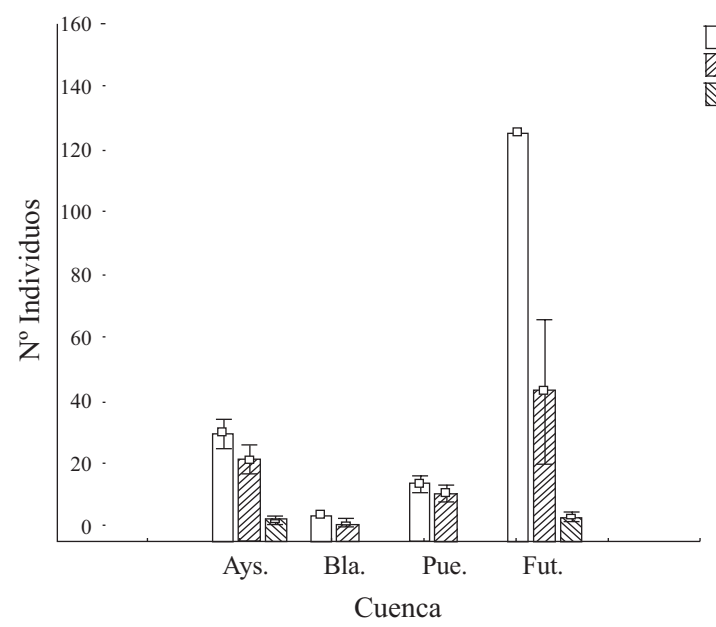

b)
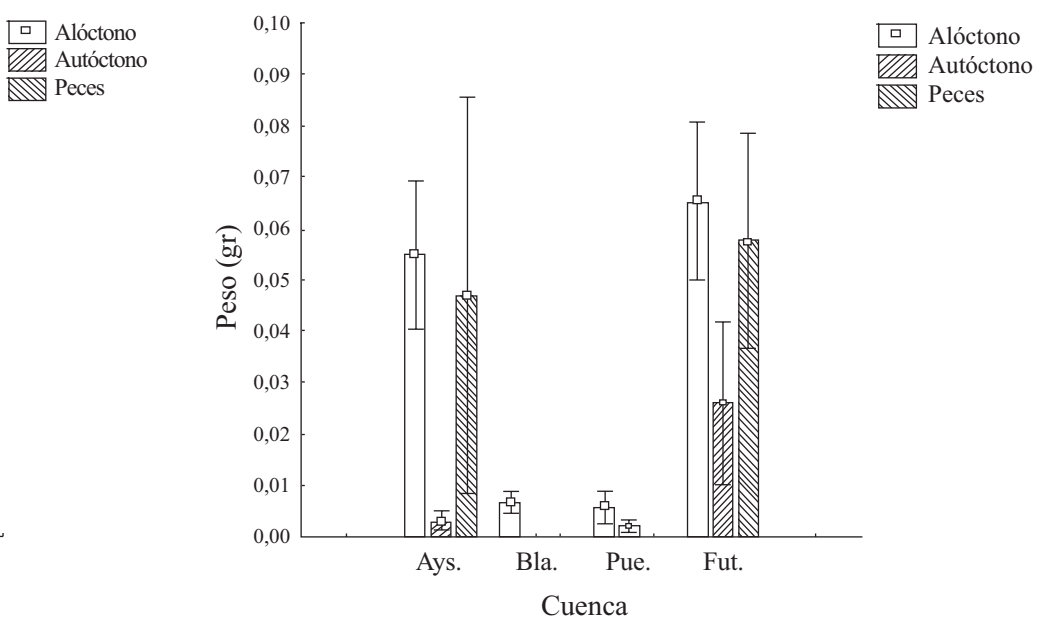

Figura 6. a) Aporte numérico promedio ( \pm 1 error estándar) y b) Aporte gravimétrico promedio ( \pm 1 error estándar) de los ítemes Alóctono, Autóctono y Peces por cuenca. Ays: Aysén, Bla: Blanco, Pue: Puelo, Fut: Futaleufú.

Figure 6. a) Average numerical contribution and b) Average gravimetric contribution of Allochthonous, Autochthonous and Fish prey items by basin, in which the standard error is used as a measure of deviation. Ays: Aysén, Bla: Blanco, Pue: Puelo, Fut: Futaleufú.

TABla 3. Promedio de similitud dietaria de individuos menores a 1 año $(0+)$ y de 1 año de edad (1+) y disimilitud promedio entre ellos (en negrita). Se muestra la contribución de los principales ítemes presa a la similitud intragrupo y a la disimilitud inter grupos, basado en el análisis SIMPER de una vía para la composición numérica de la dieta.

TABLE 3. Average dietary similarity of individuals younger than ( $0+$ ) 1 year old and older than (1+), and between them (in bold) plus the contribution of major prey items of these similarities one-way SIMPER analysis based on the numerical composition of the diet.

\begin{tabular}{|c|c|c|c|}
\hline \multirow[b]{2}{*}{ Ítemes } & \multicolumn{2}{|c|}{$\begin{array}{c}\text { Similitud media } \\
\text { y Contribución (\%) }\end{array}$} & \multirow{2}{*}{$\begin{array}{c}\text { Disimilitud media y Contribución (\%) } \\
\text { Edad } 0+\text { v/s } 1+\mathbf{8 2 , 7 1} \\
\end{array}$} \\
\hline & Edad $0+\mathbf{2 4 , 4 2}$ & Edad 1+ 26,62 & \\
\hline Chironomidae (Larva) & & $5,24(19,70)$ & $6,83(8,26)$ \\
\hline Leptophlebiidae (Adulto) & & $7,27(27,33)$ & $7,64(9,24)$ \\
\hline Trichoptera (Adulto) & $8,66(35,44)$ & $3,38(12,71)$ & $10,63(12,85)$ \\
\hline Chironomidae (Adulto) & $4,88(19,97)$ & & \\
\hline Trichoptera (Pupa) & $2,28(9,34)$ & & \\
\hline Peces (Galaxia sp.) & $1,09(4,1)$ & & $2,89(3,5)$ \\
\hline
\end{tabular}




\section{DISCUSIÓN}

Los intentos realizados a partir del año 1872 por introducir especies de importancia comercial a la Patagonia chilena (Basulto 2003), sumado a las características de la íctiofauna dulceacuícola nativa, de baja diversidad, alto grado de endemismo y baja resistencia a la invasibilidad (Cussac et al. 2004) han ayudado a que especies de salmónidos hayan establecido poblaciones autosustentables. Ello representa una amenaza aún escasamente evaluada para las especies nativas (Soto et al. 2006, Soto et al. 2007, Habit et al. 2006, Habit et al. 2010). La introducción de O. tshawytscha fue estudiada por Correa \& Gross (2007) desde la perspectiva de su invasión a escala geográfica en Chile y por Pascual et al. (2002) en cuanto a los efectos potenciales de las especies exóticas. La presencia de poblaciones juveniles de Chinook puede generar diferentes efectos sobre las comunidades ícticas. Así por ejemplo, se ha reportado que en su etapa juvenil los chinook del tipo oceánico presentan un comportamiento territorial agresivo en agua dulce (Healey 1991). Ello puede generar una interacción negativa por competencia por el espacio entre las especies nativas y los demás salmónidos asilvestrados por más de 100 años en ríos y lagos patagónicos como las truchas café y arcoíris. En este estudio nos centramos en el potencial efecto de depredación que podrían estar ejerciendo las poblaciones de juveniles de salmón Chinook durante su etapa dulceacuícola. En primer lugar, determinamos que existen poblaciones stream-type (sensu Healey 1991, Ruiz \& Marchant 2004) en ríos de la Patagonia chilena, y no sólo poblaciones ocean-type, tal como lo sugirieron Soto et al. (2007) y en segundo lugar, determinamos que pueden coexistir cohortes de 0 y 1 año de edad en algunos de estos sistemas. Dada la falta de información respecto a la determinación del momento de emigración al mar de individuos de las formas ocean-type y stream-type asilvestradas, no es posible determinar el tiempo de coexistencia de estas dos cohortes en los sistemas patagónicos.

Los salmónidos son conocidos por sus hábitos voraces y su efecto sobre la fauna nativa, produciendo, por ejemplo, el aumento de los niveles de piscivoría en ríos y lagos (Ortiz-Sandoval 2006, Piedra 2006). Se ha descrito que los juveniles de Chinook presentan una dieta dominada principalmente por macroinvertebrados bentónicos (Jonsson et al. 1999), organismos autóctonos como larvas de dípteros, larvas y pupas de chironómidos y copépodos (Limm \& Marchetti 2009). Koheler et al., (2006) menciona que en el lago Washington, entre los meses de febrero y mayo, los juveniles silvestres de salmón Chinook consumen sobre todo presas epibénticas, principalmente pupas de chironómidos (Diptera); mientras que en junio cambian a una dieta dominada por el plancton, en particular Daphnia spp. Tal información, proveniente de sistemas acuáticos de donde la especie es nativa (desde California hasta Alaska, y las costas asiáticas del Pacífico norte sensu Drummond 1982), no concuerda con lo encontrado en las cuencas de Aysén y Futaleufú, donde los ítemes alimentarios de mayor aporte numérico correspondieron a organismos de origen alóctono. En tanto, el mayor aporte en biomasa lo compartieron ítemes alóctonos y peces nativos galáxidos. Resulta destacable que juveniles de Chinook tanto de edad 0+ como 1+ presentaron peces en sus estómagos, lo cual estuvo determinado principalmente por la talla. Entonces, juveniles que alcanzan tallas sobre $8,5 \mathrm{~cm}$ LT, aún cuando no alcancen un año de edad, adquieren hábitos ictiófagos y depredan sobre galáxidos nativos. También es relevante que este comportamiento se manifiesta tanto en ríos (cuencas del Aysén) como en lagos, lo cual indica que la piscivoría en juveniles de Chinook está modulada por la oferta alimentaria (presencia de peces) y por la talla. Concluimos, entonces, que los juveniles de salmón Chinook han ingresado a los ecosistemas dulceacuícolas patagónicos como un nuevo depredador con hábitos ictiófagos; principalmente sobre especies de galáxidos nativos, implicando una amenaza más a esta fauna altamente amenazada.

\section{AGRADECIMIENTOS}

Agradecemos a Jorge González, Juan José Ortiz, Roberto Cifuentes, Ignacio Rudolph y Mónica Montory por colaborar en la captura de los peces. A Mark Belk por realizar la doble lectura de otolitos y a los proyectos: FONDECYT $\mathrm{N}^{\mathrm{o}} 1080294$ "Biotransport of Persistent Organic Pollutants to Rivers and Rivers and Lakes in the Northern Patagonia (Aysén Region) Chile: The role of migrating salmons"; FONDECYT N 1080082 "Latitudinal and Local Patterns of Diversity in the Native and non-Native Lacustrine Fish Fauna of The Chilean Patagonia” y al Proyecto National Geographic Society (NGS 8168-07) "Phylogeography, speciation and conservation genetics of Patagonian Native Fishes".

\section{BIBLIOGRAFÍA}

Aigo, J., Cussac, V., Peris, S., Ortubay, S., Gómez, S., López, H., Gross, M., Barriga, J. \& Battini, M. 2008. Distribution of introduced and native fish in Patagonia (Argentina): patterns and changes in fish assemblages. Reviews in Fish Biology and Fisheries Volume 18: 387-408.

BAsulto, S. 2003. El largo viaje de los salmones: una crónica olvidada, Propagación y cultivo de especies acuáticas en Chile. Maval Limitada Editores, Santiago, Chile. 102 pp.

Bray, J.R. \& CuRTIS, JT. 1957. An ordination of the upland forest communities of southern Wisconsin. Ecological Monographs 27: 325-349.

Campos, H, G. Dazarola, B. Dyer, L. Fuentes, J. Gavilán, L Huaquin, G. Martínez, R. Menéndez, G. Pequeño, F. 
Ponce, V. Ruiz, W. Sielfeld, D. Soto, R. Vega \& Vila, I. 1998. Categorías de conservación de peces nativos de aguas continentales de Chile. Boletín del Museo Nacional de Historia Natural (Chile) 47: 101-222.

Clarke, K.R., R.N. Gorley. 2005. PRIMER v.6: User Manual / Tutorial. PRIMER-E Ltda, Playmouth, UK.

Clarke, K.R., R.M. Warwick, P.J. Somerfield \& R.N. Gorley. 2005. Changer in marine communities: an approach to statistical analysis and interpretation, 3rd edn. PRIMER-E Ltada, Playmouth, UK.

Correa, C. \& Gross, M. R. 2007. Chinook salmon invade southern South America. Biological Invasions 10(5): 615.

Cussac, V., Ortubay, S., Iglesias, G., Milano, D., Lattuca, M., Barriga, J., Battini, M., \& Gross, M. 2004. The distribution of South American galaxiid fishes: the role of biological traits and post glacial history. Journal of Biogeography 31: 103-122.

Drummond, S. 1982. The Salmon Handbook, the life and cultivation of fishes of the salmon family. André Deutsch Limited. London. 242 pp.

Fulton, T. W. 1902. The rate of growth of fishes. 20th Annual Report of the Fishery Board of Scotland 3: 326-446.

Habit, E., Dyer, B., \& I. Vila. 2006. Estado del conocimiento de los peces dulceacuícolas de Chile. Gayana Zoología 70 (1): 100-113.

Habit, E., Piedra, P., Ruzzante, D., Walde, S., Belk, M., Cussac, V., González, J., \& Colin, N. 2010. Changes in the distribution of native fi shes in response to introduced species and other anthropogenic effects. Global Ecology \& Biogeography 19 (5): 697-710.

HEALEY, M.C. 1991. Lifehistory of ChS(Oncorhynchus tshawytscha). In: Groot C., Margolis L. (eds.) Pacific salmon life histories. UBC Press, Vancouver, BC, 311-396 pp.

Jonsson, N., Naesje, T.F., Jonsson B., Saksgård R. \& Sandlund O. 1999. The influence of piscivory on life history traits of Brown trout. Journal of Fish Biology. 55: 1129-1141.

Koheler, M., Fresh, K., Beauchamp, D., Condell, J., Simenstad, C., \& Seiler, D. 2006. Diet and Bioenergetics of LakeRearing Juvenile Chinook Salmon in Lake Washington. Transactions of the American Fisheries Society 135:15801591.

Limm, M. \& MarchetTI, M. 2009. Juvenile Chinook salmon (Oncorhynchus tshawytscha) growth in off-channel and main-channel habitats on the Sacramento River, CA using otolith increment widths. Environ Biol Fish 85:141-151.

Macchi, P.J., Cussac V., Alonso M. \& Denegri M. 1999. Predation relationships between salmonids and the native fish fauna in lakes and reservoirs in northern Patagonia. Ecology of Freshwater Fish, 8: 227-236.

McIntosh, A.R. 2000. Habitat and size-related variations in exotic trout impacts on native galaxiid fishes in New Zealand streams. Canadian Journal of Fisheries and Aquatic Sciences, 57: 2140-2151.

Milano, D., Cussac, V., Macchi, P., Ruzzante, D., Alonso, M., Vigliano, P. \& Denegri, M. 2002. Predator associated morphology in Galaxias platei in Patagonian lakes. Journal of Fish Biology, 61:138-156.

Milano, D., Ruzzante, D., Cussac, V., Macchi, P., Ferriz, R., Barriga, J., Aigo, J., Lattuca, M. \& Walde, S. 2006. Latitudinal and ecological correlates of morphological variation in Galaxias platei (Pisces, Galaxiidae) in Patagonia. Biological Journal of the Linnean Society 87 (1) 69-82.

Naiman, R.J., Bilby, R.E., De Schindler \& Hedfield, J.M. 2002. Pacific salmon, nutrients and the dynamics of freshwater and riparian ecosystems. Ecosystems 5: 399-417.

Neilson, J.D. \& Geen G. H. 1982. Otolith of chinook salmon (Oncorhynchus tshawytscha): daily growth increments and factors influencing their production. Canadian Journal of Fisheries and aquatic Sciences 39:1340-7.

Ortiz-Sandoval J. 2006. Estructura trófica de la ictiofauna de la zona limnética y profunda de lagos andino-patagónicos de la Región de Aysén: niveles de piscivoría asociados a salmonídeos y especies nativas. Tesis para optar al título de Biólogo. Universidad de Concepción. Chile. 59 pp.

Pascual, M., Macchi, P., Urbanski, J., Marcos, F., Riva-Rossi, C., Novara, M. \& Dell'Arciprete, P. 2002. Evaluating potential effects of exotic freshwater fish from incomplete species presence-absence data. Biological Invasions 4: 101-113.

PIEDRA, P. 2006. Estructura comunitaria de ictiofauna nativa e introducida de ríos y lagos de la Patagonia chilena (XI Región Aysén, Chile). Tesis para optar al título de Biólogo Marino. Universidad de Concepción. Chile. 59 pp.

Ruiz, H. \& Marchant, M. 2004. Ictiofauna de aguas continentales chilenas. Dirección de Docencia. Universidad de Concepción. 356 pp.

Soто, D. 1997. Evaluación de salmónidos de vida libre existentes en las aguas interiores de las regiones X y XI. Informe Técnico, Fondo de Investigación Pesquera, Subsecretaría de Pesca, Chile, FIO 95-141.

Soto, D., Jara, H.F. \& Moreno, C.A. 2001. Escaped salmon in the Chiloé and Aysén inner seas, southern Chile: facing ecological and social conflicts. Ecological Applications 11: $1750-1762$

Soto, D., Arismendi, I., GonzÁlez, J., Guzmán, E., Sanzana, J., JARA, F., JARA, C. \& LARA, A. 2006. Southern Chile, trout and salmon country: conditions for invasion success and challenges for biodiversity conservation. Revista Chilena de Historia Natural 79: 97-117.

Soto, D., Arismendi, I., Prinzio, C. \& JARA, F. 2007. Establishment of Chinook zalmon (Oncorhynchus tshawytscha) in Pacific basins of southern South America and its potential ecosystem implications. Revista Chilena de Historia Natural. 80: 81-98.

Unwin, M.J. \& QuinN, T.P. 1993. Homing and straying patterns of ChS (Oncorhynchus tshawytscha) from a New Zealand hatchery: spatial distribution of strays and effects of release date. Canadian Journal of Fisheries and aquatic Sciences 50:1168-1175.

Recibido: 15.06.10

Aceptado: 06.04.11 